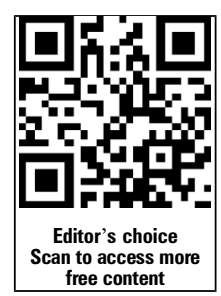

${ }^{1}$ Division of Neonatology, Center of Maternal-Fetal, Neonatal and Reproductive Medicine, National Center for Child Health and Development, Tokyo, Japan

${ }^{2}$ Department of Developmental Social Medicine, National Research Institute for Child Health and Development, Tokyo, Japan

${ }^{3}$ Center of Maternal-Fetal, Neonatal and Reproductive Medicine, National Center for Child Health and Development, Tokyo, Japan

\section{Correspondence to} Dr Kenji Hishikawa, Division of Neonatology, Center of Maternal-Fetal, Neonatal and Reproductive Medicine, National Center for Child Health and Development, 2-10-1 Okura Setagaya-ku, Tokyo 157-8535, Japan; hishikawa.k1234@gmail.com

Received 14 November 2014 Revised 11 March 2015 Accepted 12 March 2015 Published Online First 8 April 2015
CrossMark

To cite: Hishikawa $K$, Goishi K, Fujiwara T, et al. Arch Dis Child Fetal Neonatal Ed 2015;100 F382-F387.

\title{
Pulmonary air leak associated with CPAP at term birth resuscitation
}

\author{
Kenji Hishikawa, ${ }^{1}$ Keiji Goishi, ${ }^{1}$ Takeo Fujiwara, ${ }^{2}$ Masao Kaneshige, ${ }^{1}$ Yushi Ito, ${ }^{1}$ \\ Haruhiko Sago ${ }^{3}$
}

\begin{abstract}
Objective The Japan Resuscitation Council (JRC)

Guidelines 2010 for neonatal resuscitation introduced continuous positive airway pressure (CPAP) in delivery room. The present study evaluated the effect of CPAP for pulmonary air leak at term birth.

Design, setting and patients This retrospective single-centre study used the data of term neonates who were born without major congenital anomalies at our

\section{What is already known on this topic}

The International Liaison Committee on Resuscitation Consensus 2010 and the Japan Resuscitation Council (JRC) Guidelines 2010 introduced continuous positive airway pressure (CPAP) in delivery room. Whether CPAP increases pulmonary air leaks at term birth remains unclear.
\end{abstract} centre between 2008 and 2009, and between 2011 and 2012.

Interventions Resuscitation according to the JRC Guidelines 2010.

Main outcome measures We examined the association between the JRC Guidelines 2010, CPAP by face mask and pulmonary air leak.

Results A total of 5038 infants were analysed. The frequency of CPAP by face mask increased after the update of the JRC Guidelines in 2010 (1.7\% vs $11.1 \% ; p<0.001)$. Pulmonary air leak increased at early term (37 weeks: $1.0 \%$ vs $3.5 \%, p=0.02 ; 38$ weeks: $0.7 \%$ vs $2.2 \%$, $p=0.02$ ). While adjusting for confounders, the JRC Guidelines 2010 was associated with pulmonary air leak in early-term neonates (37 weeks: adjusted OR (aOR) 4.37;

$95 \% \mathrm{Cl} 1.40$ to $17.45 ; 38$ weeks: aOR $2.80 ; 95 \% \mathrm{Cl} 1.04$ to 8.91), but this association disappeared while adjusting for face mask CPAP additionally (37 weeks: aOR 1.90; $95 \% \mathrm{Cl} 0.47$ to 8.71 ; 38 weeks: aOR 1.66; $95 \% \mathrm{Cl} 0.54$ to 5.77 ).

Conclusions Following the update of the JRC guidelines on neonatal resuscitation, we observed an increased use of CPAP via face mask, which was associated with a higher prevalence of pulmonary air leak in early-term neonates in our centre.

\section{INTRODUCTION}

Respiratory distress is common in newborns. One of the main aetiologies of neonatal respiratory distress is pulmonary air leak including pneumothorax and pneumomediastinum. Pulmonary air leak is more likely to occur in the neonatal period than at any stage of life. Data on the prevalence of pulmonary air leak primarily reflect the high incidence rate of pneumothorax. Symptomatic pneumothorax occurs in $0.08 \%$ of all live births. ${ }^{1}$

An estimated $5-10 \%$ of newborns require resuscitation at birth. ${ }^{2}{ }^{3}$ To that end, the International Liaison Committee on Resuscitation (ILCOR) released a consensus for neonatal cardiopulmonary resuscitation (NCPR) in 2005 and $2010 .{ }^{4}$ Based on these two consensuses, the Japan Resuscitation Council (JRC) released the guidelines for NCPR in 2007 and an update in 2010. ${ }^{6}$ Both the JRC

\section{What this study adds}

The update of the JRC Guidelines in 2010 increased the frequency of CPAP by face mask performed in term neonates.

- CPAP led to a higher prevalence of pulmonary air leak in early-term neonates.

Guidelines 2010 and the ILCOR Consensus 2010 recommended a low-oxygen approach and continuous positive airway pressure (CPAP) in delivery room for newborn resuscitation to recruit lung volume and avoid oxygen toxicity.

CPAP is useful for adequate functional residual capacity and to maintain open alveoli and distal airways. ${ }^{7}$ However, there is no evidence to support or refute the use of CPAP in delivery room for term neonates. ${ }^{5}$ The update of the JRC Guidelines and the ILCOR Consensus in 2010 is thought to have caused an increase in the use of CPAP in delivery rooms worldwide. Morley $e t \mathrm{al}^{8}$ showed that CPAP increased the prevalence of pneumothorax of preterm infants. However, little is known whether CPAP induces pulmonary air leak in term newborns or not. The present study evaluates the effects of CPAP on pulmonary air leak at term birth following the update of the JRC guidelines in 2010.

\section{METHODS}

\section{Study design}

We organised a retrospective, single-centre study at our hospital, the National Center for Child Health and Development, a tertiary perinatal centre in Tokyo, Japan. Our hospital also cares for low-risk pregnant women and infants.

\section{Study sample and data collection}

Approximately 1700 births occurred annually at our hospital. In order to evaluate the prevalence of pulmonary air leak at term birth according to the update of the JRC Guidelines in 2010, we used the 
data of term neonates who were born at our hospital between 2008 and 2009 (defined as 'group 1'), as well as those who were born between 2011 and 2012 (defined as 'group 2'). Data of stillbirths, neonates with major abnormalities such as malformation syndrome, chromosome disease, central nerve disease, thoracic disease, congenital heart disease, gastrointestinal disease, renal disease and skeletal disease or other organ diseases, and births with insufficient records were excluded.

We extracted detailed maternal and neonatal information from electronic medical records, electronic labour database, and maternal and neonatal discharge summaries. Prenatal history, together with labour and delivery progression, was recorded by trained obstetricians and midwives on delivery charts linked to the electronic labour database.

\section{Maternal, infantile and resuscitation variables}

For analysis, the following maternal variables were used: maternal age, multipara, multiple births, premature rupture of membranes, meconium staining of the amniotic fluid, abruptio placentae, labour induction, epidural labour analgesia and mode of delivery. Infantile variables included gestational weeks, gender, birth weight, low birth weight $(<2500 \mathrm{~g})$, low Apgar score at $1 \mathrm{~min}(\leq 7)$, low Apgar score at $5 \mathrm{~min}(\leq 7)$ and the length of hospital stay. Resuscitation variables included oxygen treatment, face mask CPAP and bag-and-mask ventilation in delivery room.

\section{Resuscitation}

NCPR was performed according to the JRC Guidelines by doctors with certification in neonatal cardiopulmonary resuscitation (awarded by the Japan Society of Perinatal and Neonatal Medicine). Up until 2010, 100\% oxygen was used for NCPR. After the release of the JRC Guidelines 2010, oxygen blended with air under the assessment of oximetry was used instead. The JRC Guidelines 2010 described the use of supplemental oxygen as follows: "For babies born at term it is best to begin resuscitation with air rather than $100 \%$ oxygen. Administration of supplementary oxygen should be regulated by blending oxygen and air, and the concentration delivered should be guided by oximetry." In this study, oxygen treatment was defined as oxygen or blended oxygen with free flow, face mask CPAP or bag-and-mask ventilation. The JRC Guidelines 2010 introduced CPAP and described its use as follows: "If the baby has labored breathing and cyanosis, you should use pulse oximeter and may assist the baby's breathing by CPAP with room air or may give him supplemental oxygen." Prior to the 2010 Guidelines, face mask CPAP in babies was performed with continued laboured breathing or cyanosis under supplemental oxygen. In both periods, face mask CPAP commenced at a pressure of $5-6 \mathrm{~cm} \mathrm{H}_{2} \mathrm{O}$, and bag-and-mask ventilation was performed with a starting peak inspiratory pressure of $20 \mathrm{~cm} \mathrm{H}_{2} \mathrm{O}$ and an end expiratory pressure of $5-6 \mathrm{~cm} \mathrm{H}_{2} \mathrm{O}$. The peak inspiratory pressure was controlled according to heart rate and rising of the chest. No CPAP beyond $6 \mathrm{~cm} \mathrm{H}_{2} \mathrm{O}$ was performed, neither was sustained inflations during the resuscitation. The resuscitation device used was the same in both period, and so was the face mask that has a flow-inflating bag with flow-control valve and pressure manometer.

\section{Outcome and definitions}

The primary outcome for consideration was neonatal pulmonary air leak. Pulmonary air leak included pneumothorax and pneumomediastinum within $24 \mathrm{~h}$ of birth accompanied with dyspnoea. Pulmonary air leak was diagnosed by neonatologists based on clinical findings, and the diagnosis was confirmed by radiologist using chest X-rays. Chest X-rays were performed in all cases with respiratory distress requiring treatment after resuscitation. Secondary outcomes for consideration were treatment of pulmonary air leak (neonatal intensive care unit (NICU) admission, observation only, oxygen therapy only, mechanical ventilation and thoracentesis) and death related to pulmonary air leak. Oxygen therapy only was defined as supplemental oxygen with oxygen head box or nasal cannula in newborn nursery or NICU after resuscitation in delivery room.

\section{Statistical analysis}

First, we compared maternal and infantile variables of both groups. Further, we compared resuscitation variables of both groups at every gestational week. We compared categorical variables of both groups with the $\chi^{2}$ test when appropriate; otherwise, the Fisher's exact test was used. We compared continuous variables of both groups with Student $t$ test or Wilcoxon rank-sum test on the basis of the distribution.

Next, we analysed the association between the update of the JRC Guidelines and the resuscitation variables at each gestational week to confirm whether the guidelines influenced oxygen treatment, face mask CPAP and bag-and-mask ventilation in the delivery room. To adjust for confounders, we used multiple logistic regression models. Maternal and infantile variables with $p$ values $<0.2$ for the association between each group's variables (maternal age, multipara, multiple births, abruptio placentae, labour induction, epidural labour analgesia, low Apgar score at $5 \mathrm{~min}$ of $\leq 7)$ were included, and the confounders included meconium staining of the amniotic fluid, caesarean section and low birth weight $(<2500 \mathrm{~g})$. Low Apgar score at $1 \mathrm{~min}$ was excluded because of strong association with low Apgar score at $5 \mathrm{~min}$.

In addition, we compared the prevalence of pulmonary air leak and each type of pulmonary air leak with frequencies of treatment (with the same secondary outcomes described above and death related to pulmonary air leak) in both groups. Next, we analysed the association between pulmonary air leak and the update of the JRC Guidelines at each gestational week. At first, we did not include any of the resuscitation variables (oxygen treatment, face mask CPAP and bag-and-mask ventilation in delivery room) as confounders because we considered resuscitation variables to be intermediate variables between update of the JRC Guidelines and pulmonary air leak. Thereafter, we included each resuscitation variable as a confounder to confirm which resuscitation variables were intermediate variables that influenced pulmonary air leak.

We estimated adjusted ORs (aORs) and 95\% CIs. A two-sided $\mathrm{p}<0.05$ was considered significant for all tests, and all statistical analyses were performed using JMP software (V.10, SAS Institute).

\section{RESULTS}

Study sample

The study sample flow is shown in figure 1. A total of 5038 infants were analysed, of which 2483 infants were included in group 1 and 2555 infants were included in group 2 . The maternal and infantile baseline demographic and clinical characteristics of each group are shown in table 1. Group 2 was older, had higher incidence of epidural labour analgesia, low Apgar score at $1 \mathrm{~min}(\leq 7)$ and $5 \mathrm{~min}(\leq 7)$, and longer hospital stay, but had lower incidence of abruptio placentae, labour induction and normal vaginal delivery.

\section{Characteristics of resuscitation}

In group 2, oxygen treatment was decreased significantly at every gestational week except week 37 in univariate and 


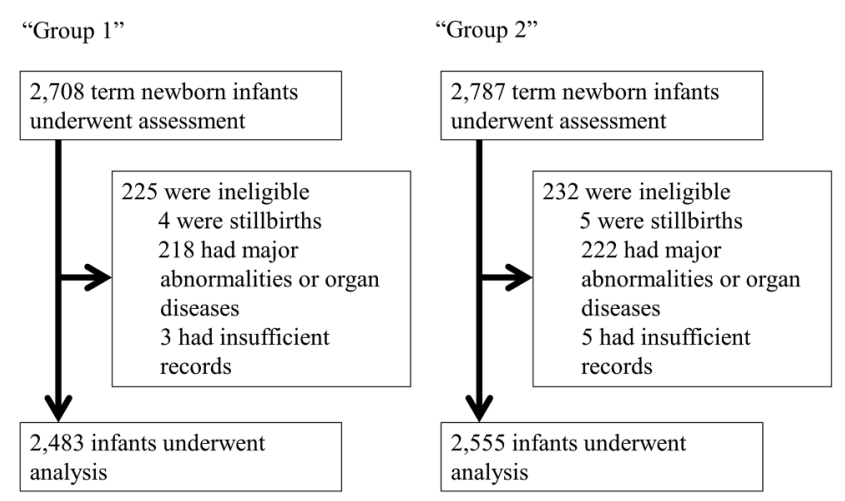

Figure 1 Study sample flow. Group 1: term neonates born at our hospital between 2008 and 2009. Group 2: term neonates born at our hospital between 2011 and 2012.

multivariate analysis (table 2). Face mask CPAP increased significantly at every gestational week in univariate and multivariate analysis. Bag-and-mask ventilation was not different at any gestational week except week 38 in multivariate analysis.

\section{Prevalence and severity of pulmonary air leak}

The frequency of chest X-rays and the prevalence of pulmonary air leak increased significantly in group 2 compared with group 1 (chest X-rays: group 1, 8.1\% vs group 2, 17.0\%; $\mathrm{p}<0.001$; pulmonary air leak: group $1,1.0 \%$ vs group $2,1.8 \% ; \mathrm{p}=0.01$ ) (table 3). The frequencies of each treatment for infants with pulmonary air leak were not different between two groups, except oxygen therapy only (group $1,0.8 \%$ vs group 2, $1.5 \%$; $\mathrm{p}=0.02)$. No neonate expired because of pulmonary air leak.

\section{Characteristics of pulmonary air leak}

Prevalence of pulmonary air leak increased significantly in earlyterm newborns ( 37 weeks, $1.0 \%$ vs $3.5 \%, \mathrm{p}=0.02 ; 38$ weeks: $0.7 \%$ vs $2.2 \% ; \mathrm{p}=0.02$ ) (table 4 ). The updated JRC Guideline (ie, group 2) was associated with pulmonary air leak in early- term newborns (37 weeks: aOR $4.37 ; 95 \%$ CI 1.40 to 17.45 ; 38 weeks: aOR $2.80 ; 95 \%$ CI 1.04 to 8.91$)$. These associations disappeared while adjusting for face mask CPAP (37 weeks: aOR 1.90 ; $95 \%$ CI 0.47 to 8.71 ; 38 weeks: aOR 1.66 ; $95 \%$ CI 0.54 to 5.77 ), but did not disappear while adjusting for oxygen treatment or bag-and-mask ventilation.

\section{DISCUSSION}

The JRC Guidelines 2010 and the ILCOR Consensus 2010 introduced CPAP in delivery room based on studies of preterm infants. ${ }^{8-11}$ Prior to our study, we expected the frequency of face mask CPAP in delivery room to be increased at term birth. Indeed, our study has proven this hypothesis (table 2). As well, we found a significant increase of mild pulmonary air leak after the update of the JRC Guidelines in 2010 (table 3). Furthermore, face mask $\mathrm{CPAP}$ in the delivery room increased the prevalence of pulmonary air leak in early-term neonates (table 4).

The association between the update of the guidelines and pulmonary air leak in early-term neonates is suspected to be due to the modification in the description of resuscitation. This association disappeared while adjusting for CPAP by face mask, but did not disappear while adjusting for oxygen treatment or bag-and-mask ventilation (table 4). Hence, we can deduce that CPAP by face mask was an intermediate variable but not oxygen treatment and bag-and-mask ventilation in the association between the update of the guidelines in 2010 and pulmonary air leak in early-term newborns. In other words, face mask CPAP did increase pulmonary air leak in early-term newborns but oxygen treatment and bag-and-mask ventilation did not.

It is a controversial topic whether CPAP in delivery room increases prevalence of pulmonary air leak; te Pas and Walther ${ }^{11}$ reported a lowered prevalence. Morley et $a l^{8}$ reported an increased prevalence, while Finer et $a l^{12}$ did not observe a significant difference at all. All of these studies included preterm infants. To the best of our knowledge, our study is the first report on term newborns that revealed face mask CPAP in delivery room induces pulmonary air leak at early term.

Table 1 Maternal and infantile baseline demographic and clinical characteristics of each group

\begin{tabular}{|c|c|c|c|}
\hline Characteristics & Group 1, no. (\%) ( $\mathrm{N}=2483)$ & Group 2, no. (\%) (N=2555) & $p$ Value \\
\hline \multicolumn{4}{|l|}{ Maternal baseline } \\
\hline Maternal age, mean (SD), years & $34.5(4.1)$ & $35.3(4.4)$ & $<0.001$ \\
\hline Multipara & $1083(43.6)$ & $1055(41.2)$ & 0.09 \\
\hline Multiple births & $96(3.8)$ & $126(4.9)$ & 0.06 \\
\hline Premature rupture of membranes & $375(15.1)$ & $395(15.4)$ & 0.72 \\
\hline Meconium staining of the amniotic fluid & $362(14.5)$ & $403(15.7)$ & 0.23 \\
\hline Abruptio placentae & $37(1.4)$ & $13(0.5)$ & $<0.001$ \\
\hline Labour induction & $523(21.0)$ & $362(14.1)$ & $<0.001$ \\
\hline Epidural labour analgesia & $1016(40.9)$ & $1153(45.1)$ & 0.002 \\
\hline Normal vaginal delivery & $1411(56.8)$ & $1367(53.5)$ & 0.01 \\
\hline Operative vaginal delivery & 409 (16.4) & 457 (17.8) & 0.18 \\
\hline Planed Caesarean section & $433(17.4)$ & 485 (18.9) & 0.15 \\
\hline Emergent Caesarean section & $228(9.1)$ & $246(9.6)$ & 0.58 \\
\hline \multicolumn{4}{|l|}{ Infantile baseline } \\
\hline Gestational week, mean (SD), weeks & $38.7(1.1)$ & $38.7(1.1)$ & 0.93 \\
\hline Male sex & $1280(51.5)$ & $1294(50.6)$ & 0.52 \\
\hline Birth weight, mean (SD), g & $3003(374)$ & $3000(386)$ & 0.80 \\
\hline$<2500 \mathrm{~g}$ & $205(8.2)$ & $227(8.8)$ & 0.42 \\
\hline Low Apgar score at $1 \min (\leq 7)$ & $180(7.2)$ & $234(9.1)$ & 0.01 \\
\hline Low Apgar score at $5 \min (\leq 7)$ & $24(0.9)$ & $50(1.9)$ & 0.003 \\
\hline Hospitalized days, mean (SD), days & $8.14(2.58)$ & $8.17(3.32)$ & 0.03 \\
\hline
\end{tabular}


Table 2 Characteristics of resuscitation in delivery room

\begin{tabular}{|c|c|c|c|c|c|c|c|}
\hline Gestational weeks & Group 1, n/N (\%) & Group 2, n/N (\%) & $\mathrm{p}$ Value & $\begin{array}{l}\text { Group 1, } \\
\text { OR }(95 \% \mathrm{Cl})\end{array}$ & $\begin{array}{l}\text { Group 2, } \\
\text { OR }(95 \% \mathrm{Cl})\end{array}$ & $\begin{array}{l}\text { Group 1, } \\
\text { aOR }(95 \% \mathrm{Cl})^{*}\end{array}$ & $\begin{array}{l}\text { Group 2, } \\
\text { aOR }(95 \% \mathrm{Cl})^{*}\end{array}$ \\
\hline \multicolumn{8}{|l|}{ Oxygen treatment } \\
\hline Total & $967 / 2483(38.9)$ & $566 / 2555(22.1)$ & $<0.001$ & 1 [reference] & 0.44 (0.39 to 0.50$)$ & 1 [reference] & 0.38 (0.34 to 0.44$)$ \\
\hline 37 & $138 / 373(37.0)$ & $117 / 369(31.7)$ & 0.12 & 1 [reference] & $0.79(0.58$ to 1.07$)$ & 1 [reference] & $0.72(0.52$ to 1.00$)$ \\
\hline 38 & $278 / 654(42.5)$ & $151 / 713(21.8)$ & $<0.001$ & 1 [reference] & $0.36(0.28$ to 0.46$)$ & 1 [reference] & 0.32 (0.24 to 0.41$)$ \\
\hline 39 & 247/737 (33.5) & 139/723 (19.2) & $<0.001$ & 1 [reference] & 0.47 (0.37 to 0.59$)$ & 1 [reference] & 0.42 (0.32 to 0.54$)$ \\
\hline 40 & $235 / 549(42.8)$ & $111 / 568(19.5)$ & $<0.001$ & 1 [reference] & 0.32 (0.24 to 0.42$)$ & 1 [reference] & $0.28(0.20$ to 0.37$)$ \\
\hline 41 & 69/170 (40.5) & 48/182 (26.3) & 0.004 & 1 [reference] & 0.52 (0.33 to 0.82 ) & 1 [reference] & $0.41(0.25$ to 0.67$)$ \\
\hline \multicolumn{8}{|l|}{ Face mask CPAP } \\
\hline Total & 43/2483 (1.7) & $285 / 2555$ (11.1) & $<0.001$ & 1 [reference] & 7.12 (5.20 to 9.99$)$ & 1 [reference] & 7.02 (5.08 to 9.93 ) \\
\hline 37 & 6/373 (1.6) & 63/369 (17.0) & $<0.001$ & 1 [reference] & 12.59 (5.82 to 32.98$)$ & 1 [reference] & 12.48 (5.56 to 33.78$)$ \\
\hline 38 & 10/654 (1.5) & 74/713 (10.3) & $<0.001$ & 1 [reference] & 7.45 (4.00 to 15.49 ) & 1 [reference] & 7.80 (4.12 to 16.40$)$ \\
\hline 39 & $8 / 737(1.0)$ & 70/723 (9.6) & $<0.001$ & 1 [reference] & 9.76 (4.95 to 22.16$)$ & 1 [reference] & 10.16 (5.04 to 23.48$)$ \\
\hline 40 & $14 / 549(2.5)$ & $56 / 568(9.8)$ & $<0.001$ & 1 [reference] & 4.17 (2.36 to 7.89$)$ & 1 [reference] & 4.43 (2.38 to 8.84 ) \\
\hline 41 & $5 / 170(2.9)$ & 22/182 (12.0) & 0.001 & 1 [reference] & $4.53(1.80$ to 13.80$)$ & 1 [reference] & 3.81 (1.44 to 12.08$)$ \\
\hline \multicolumn{8}{|c|}{ Bag-and-mask ventilation } \\
\hline Total & $58 / 2483(2.3)$ & 79/2555 (3.0) & 0.09 & 1 [reference] & 1.33 (0.94 to 1.88$)$ & 1 [reference] & $1.10(0.75$ to 1.62$)$ \\
\hline 37 & $7 / 373(1.8)$ & $12 / 369(3.2)$ & 0.23 & 1 [reference] & 1.75 (0.69 to 4.76$)$ & 1 [reference] & 2.19 (0.75 to 7.18$)$ \\
\hline 38 & $17 / 654(2.6)$ & $13 / 713(1.8)$ & 0.32 & 1 [reference] & $0.69(0.32$ to 1.43$)$ & 1 [reference] & 0.34 (0.11 to 0.89$)$ \\
\hline 39 & 18/737 (2.4) & $30 / 723(4.1)$ & 0.06 & 1 [reference] & 1.72 (0.96 to 3.18$)$ & 1 [reference] & 1.44 (0.71 to 2.97$)$ \\
\hline 40 & 13/549 (2.3) & 15/568 (2.6) & 0.77 & 1 [reference] & $1.11(0.52$ to 2.40$)$ & 1 [reference] & 0.95 (0.40 to 2.30$)$ \\
\hline 41 & 3/170 (1.7) & 9/182 (4.9) & 0.10 & 1 [reference] & 2.89 (0.84 to 13.21$)$ & 1 [reference] & 3.74 (0.89 to 25.48$)$ \\
\hline
\end{tabular}

Adjusted confounders.

*Maternal age, multipara, multiple births, abruptio placentae, meconium staining of the amniotic fluid, caesarean section, labour induction, epidural labour analgesia, low Apgar score at $5 \mathrm{~min}(\leq 7)$, low birth weight $(<2500 \mathrm{~g}) . \mathrm{aOR}$, adjusted OR; CPAP, continuous positive airway pressure.

Table 3 Prevalence and severity of each type of pulmonary air leak

\begin{tabular}{|c|c|c|c|}
\hline & Group 1, no. $(\%)(\mathrm{N}=2483)$ & Group 2, no. $(\%)(\mathrm{N}=2555)$ & p Value \\
\hline Chest X-rays & $201(8.1)$ & $436(17.0)$ & $<0.001$ \\
\hline Pulmonary air leak & $26(1.0)$ & $47(1.8)$ & 0.01 \\
\hline Pneumothorax & $22(0.8)$ & $33(1.2)$ & 0.16 \\
\hline Pneumomediastinum & $3(0.1)$ & $4(0.1)$ & 0.73 \\
\hline Pneumothorax and/or pneumomediastinum & $1(0.04)$ & $10(0.39)$ & 0.007 \\
\hline \multicolumn{4}{|l|}{ Treatment of pulmonary air leak } \\
\hline NICU admission & $14(0.5)$ & $26(1.0)$ & 0.06 \\
\hline Observation only & $1(0.04)$ & $3(0.1)$ & 0.33 \\
\hline Oxygen therapy only & $22(0.8)$ & $40(1.5)$ & 0.02 \\
\hline Mechanical ventilation & $3(0.1)$ & $4(0.1)$ & 0.73 \\
\hline Thoracentesis & $1(0.04)$ & 0 & 0.31 \\
\hline \multicolumn{4}{|l|}{ Treatment of pneumothorax } \\
\hline NICU admission & $12(0.4)$ & $17(0.6)$ & 0.39 \\
\hline Observation only & $1(0.04)$ & $2(0.08)$ & 0.58 \\
\hline Oxygen therapy only & $19(0.7)$ & $27(1.0)$ & 0.27 \\
\hline Mechanical ventilation & $2(0.08)$ & $4(0.1)$ & 0.43 \\
\hline Thoracentesis & $1(0.04)$ & 0 & 0.31 \\
\hline \multicolumn{4}{|l|}{ Treatment of pneumomediastinum } \\
\hline NICU admission & $2(0.08)$ & $2(0.08)$ & 0.97 \\
\hline Observation only & 0 & 0 & NA \\
\hline Oxygen therapy only & $2(0.08)$ & $4(0.1)$ & 0.43 \\
\hline Mechanical ventilation & $1(0.04)$ & 0 & 0.31 \\
\hline \multicolumn{4}{|c|}{ Treatment of pneumothorax and/or pneumomediastinum } \\
\hline NICU admission & 0 & $7(0.2)$ & 0.009 \\
\hline Observation only & 0 & $1(0.04)$ & 0.32 \\
\hline Oxygen therapy only & $1(0.04)$ & $9(0.3)$ & 0.01 \\
\hline Mechanical ventilation & 0 & 0 & NA \\
\hline Thoracentesis & 0 & 0 & NA \\
\hline Death related pulmonary air leak & 0 & 0 & NA \\
\hline
\end{tabular}


Table 4 Characteristics of pulmonary air leak

\begin{tabular}{lcclll}
\hline \multicolumn{1}{l}{ Prevalence of pulmonary air leak and OR } \\
\hline Gestational weeks & $\begin{array}{l}\text { Group 1, } \\
\text { n/N (\%) }\end{array}$ & $\begin{array}{l}\text { Group 2, } \\
\text { n/N (\%) }\end{array}$ & p Value & Group 1, OR (95\% Cl) & Group 2, OR (95\% Cl) \\
\hline Total & $26 / 2483(1.0)$ & $47 / 2555(1.8)$ & 0.01 & 1 [reference] & $1.77(1.10$ to 2.90) \\
37 & $4 / 373(1.0)$ & $13 / 369(3.5)$ & 0.02 & 1 [reference] & $3.36(1.17$ to 12.04) \\
38 & $5 / 654(0.7)$ & $16 / 713(2.2)$ & 0.02 & 1 [reference] & $2.97(1.15$ to 9.15$)$ \\
39 & $8 / 737(1.0)$ & $9 / 723(1.2)$ & 0.77 & 1 [reference] & $1.14(0.43$ to 3.07) \\
40 & $6 / 549(1.0)$ & $5 / 568(0.8)$ & 0.71 & 1 [reference] & $0.80(0.23$ to 2.68) \\
41 & $3 / 170(1.7)$ & $4 / 182(2.2)$ & 0.77 & 1 [reference] & $1.25(0.27$ to 6.42) \\
\hline
\end{tabular}

Adjusted OR for pulmonary air leak by update of guidelines in 2010

\begin{tabular}{|c|c|c|c|c|c|c|c|c|}
\hline \multirow[b]{2}{*}{$\begin{array}{l}\text { Gestational } \\
\text { weeks }\end{array}$} & \multicolumn{2}{|c|}{ Excluding resuscitation variables* } & \multicolumn{2}{|c|}{ Including oxygen treatment $^{\dagger}$} & \multicolumn{2}{|c|}{ Including face mask CPAP ${ }^{\ddagger}$} & \multicolumn{2}{|c|}{$\begin{array}{l}\text { Including bag-and-mask } \\
\text { ventilation }^{\S}\end{array}$} \\
\hline & $\begin{array}{l}\text { Group 1, } \\
\text { aOR }(95 \% \mathrm{CI})\end{array}$ & $\begin{array}{l}\text { Group 2, } \\
\text { aOR }(95 \% \mathrm{CI})\end{array}$ & $\begin{array}{l}\text { Group 1, } \\
\text { aOR }(95 \% \mathrm{Cl})\end{array}$ & $\begin{array}{l}\text { Group 2, } \\
\text { aOR }(95 \% \mathrm{CI})\end{array}$ & $\begin{array}{l}\text { Group 1, } \\
\text { aOR }(95 \% \mathrm{CI})\end{array}$ & $\begin{array}{l}\text { Group 2, } \\
\text { aOR }(95 \% \mathrm{CI})\end{array}$ & $\begin{array}{l}\text { Group 1, } \\
\text { aOR }(95 \% \mathrm{CI})\end{array}$ & $\begin{array}{l}\text { Group 2, } \\
\text { aOR }(95 \% \mathrm{Cl})\end{array}$ \\
\hline Total & 1 [reference] & 1.66 (1.02 to 2.76$)$ & 1 [reference] & 2.44 (1.47 to 4.13$)$ & 1 [reference] & 0.81 (0.46 to 1.44$)$ & 1 [reference] & 1.66 (1.02 to 2.77$)$ \\
\hline 37 & 1 [reference] & 4.37 (1.40 to 17.45$)$ & 1 [reference] & $5.62(1.70$ to 23.96$)$ & 1 [reference] & 1.90 (0.47 to 8.71$)$ & 1 [reference] & 4.38 (1.40 to 17.43$)$ \\
\hline 38 & 1 [reference] & $2.80(1.04$ to 8.91$)$ & 1 [reference] & $4.09(1.47$ to 13.31$)$ & 1 [reference] & $1.66(0.54$ to 5.77$)$ & 1 [reference] & 2.89 (1.07 to 9.31$)$ \\
\hline 39 & 1 [reference] & $1.17(0.42$ to 3.36$)$ & & & & & & \\
\hline 40 & 1 [reference] & 0.64 (0.17 to 2.24$)$ & & & & & & \\
\hline 41 & 1 [reference] & $1.39(0.24$ to 10.70$)$ & & & & & & \\
\hline \multicolumn{9}{|c|}{$\begin{array}{l}\text { Adjusted confounders. } \\
{ }^{*} \text { Maternal age, multipara, multiple births, abruptio placentae, } \\
\text { at } 5 \text { min }(\leq 7) \text {, low birth weight }(<2500 \mathrm{~g}) \text {. } \\
\text { †Same as * , adding oxygen treatment. } \\
\text { ‡Same as *, adding face mask CPAP. } \\
\text { §Same as *, adding bag-and-mask ventilation. } \\
\text { aOR, adjusted OR; CPAP, continuous positive airway pressure. }\end{array}$} \\
\hline
\end{tabular}

The occurrence of transient tachypnoea is generally more frequent in early-term neonates compared with term birth neonates. Sengupta et $a l^{13}$ reported that early-term neonates had a significantly higher risk for respiratory distress than full-term and late-term neonates, while Zanardo et $a l^{14}{ }^{15}$ reported that respiratory distress syndrome and pneumothorax in planned caesarean delivery at term was significantly increased in 37 and 38 weeks of gestation compared with vaginal delivery. As well, Megan et al reported that lambs that received $12 \mathrm{~cm} \mathrm{H}_{2} \mathrm{O}$ of positive end expiratory pressure died from pneumothorax. ${ }^{16}$ In all cases, a flow-inflating bag and face mask were used and not a T-piece resuscitator. We performed CPAP with 5-6 $\mathrm{cm} \mathrm{H}_{2} \mathrm{O}$ with monitoring of pressure by a manometer, but sudden crying or movement of the infant might have resulted in higher pressure in the alveoli than expected. We are aware that alveoli at earlyterm birth is more vulnerable than full-term or late-term birth, and furthermore, CPAP under respiratory distress syndrome or transient tachypnoea of newborn has an influence on the occurrence of pulmonary air leak. When CPAP is performed in newborns with transient tachypnoea, it may induce over-distension of part of the lung through check-valve mechanism of residual fluid in the peripheral airway, leading to pulmonary air leak.

Our study had several limitations. It was a retrospective singlecentre study, although we did adjust expansively to minimise the influence of confounders. We only performed chest X-rays in infants with dyspnoea, allowing us to detect pulmonary air leak with dyspnoea but not asymptomatic pulmonary air leak. Although we could not determine the true prevalence of pulmonary air leak, we believe this is not so crucial from the viewpoint of clinical practice. We did not study the long-term outcome of the infants. Therefore, we could not examine the relationship between pulmonary air leak and long-term outcome. However, the impact of increased air leak on long-term outcome might be small since there was only an increase in the number of infants with mild air leak treated with oxygen supplement. Due to the difficulty in distinguishing between mild respiratory distress syndrome, mild transient tachypnoea and mild meconium aspiration syndrome on chest X-rays and clinical courses, we did not analyse the background of pulmonary air leak in each case.

Despite the limitations, our study has several advantages. This is the first study that evaluated the association between CPAP in delivery room and pulmonary air leak in term newborns. As our centre also cares for low-risk pregnant women and infants, we were able to include data on both mild and severe cases in this study. Interpretation of all chest X-rays was done by radiologists. Hence, we were able to diagnose pulmonary air leak accurately.

In conclusion, the update of the JRC Guidelines in 2010 increased the frequency of CPAP by face mask performed in the delivery room, which led to a higher prevalence of pulmonary air leak in early-term neonates. In view of the limitations, however, we cannot firmly deduce that CPAP should not be used. Further prospective and large-scale studies are needed to confirm this finding. Nonetheless, as pulmonary air leak is a lifethreatening condition in newborns, we should pay attention to its potential occurrence when performing CPAP. It is also important to clear fluid in the airways, which can result in check-valve mechanism, before performing CPAP. As well, excessive pressure should not be applied in CPAP.

Acknowledgements We thank Dr H Mezawa, Dr T Oshima, Dr S Amari, Dr J Nagasawa, Dr I Hama, Dr Y Wada, Dr S Takahashi, Dr T Fujinaga and Dr K Tsukamoto for helpful discussions, and Ms M Fukamauchi, Dr Y Yamanobe and Dr Y Tsutsumi for data extraction. We would also like to thank Dr J Tang of the Department of Education for Clinical Research, National Center for Child Health and Development, for proofreading and editing this manuscript. 
Contributors KH conceptualised and designed the study, drafted the initial manuscript, carried out the initial analyses, and reviewed and revised the manuscript. KG conceptualised and designed the study, drafted the initial manuscript, and reviewed and revised the manuscript. TF designed the study, carried out the initial analyses and reviewed the manuscript. MK critically reviewed the manuscript. YI and $\mathrm{HS}$ critically reviewed and revised the manuscript. All authors approved the final manuscript as submitted.

\section{Competing interests None.}

Ethics approval Ethics committee of National Center for Child Health and Development, Tokyo, Japan (receipt number: 68).

Provenance and peer review Not commissioned; externally peer reviewed.

\section{REFERENCES}

1 Trevisanuto D, Doglioni N, Ferrarese P, et al. Neonatal pneumothorax: comparison between neonatal transfers and inborn infants. J Perinat Med 2005;33:449-54.

2 Palme-Kilander C. Methods of resuscitation in low-Apgar-score newborn infants--a national survey. Acta Paediatr 1992;81:739-44.

3 Saugstad OD. Practical aspects of resuscitating asphyxiated newborn infants. Eur J Pediatr 1998;157(Suppl 1):S11-15.

4 International Liaison Committee on Resuscitation. 2005 International Consensus on Cardiopulmonary Resuscitation and Emergency Cardiovascular Care Science with Treatment Recommendations. Part 7: Neonatal resuscitation. Resuscitation 2005;67:293-303.

5 Perlman JM, Wyllie J, Kattwinkel J, et al. Part 11: Neonatal resuscitation: 2010 International Consensus on Cardiopulmonary Resuscitation and Emergency Cardiovascular Care Science With Treatment Recommendations. Circulation 2010;122(16 Suppl 2):S516-38.
6 Tamura M, Wada M, Ishikawa G, et al. JRC Guidelines 2010 English version: NCPR; Neonatal Cardiopulmonary Resuscitation. Secondary JRC Guidelines 2010 English version: NCPR; Neonatal Cardiopulmonary Resuscitation. http://jrc.umin.ac.jp/pdf/ 20121011_NCPR.pdf (accessed 10 Nov 2014).

7 Wiswell TE. Resuscitation in the delivery room: lung protection from the first breath. Respir Care 2011;56:1360-7; discussion 1367-8.

8 Morley CJ, Davis PG, Doyle LW, et al. Nasal CPAP or intubation at birth for very preterm infants. N Engl J Med 2008;358:700-8.

9 Finer NN, Carlo WA, Duara $S$, et al. Delivery room continuous positive airway pressure/positive end-expiratory pressure in extremely low birth weight infants: a feasibility trial. Pediatrics 2004;114:651-7.

10 Lindner W, Vossbeck S, Hummler $\mathrm{H}$, et al. Delivery room management of extremely low birth weight infants: spontaneous breathing or intubation? Pediatrics 1999;103 (5 Pt 1):961-7.

11 te Pas $A B$, Walther FJ. A randomized, controlled trial of delivery-room respiratory management in very preterm infants. Pediatrics 2007;120:936.

12 SUPPORT Study Group of the Eunice Kennedy Shriver NICHD Neonatal ResearchNetworkFiner NN, Carlo WA, Walsh MC, et al. Early CPAP versus surfactant in extremely preterm infants. N Engl J Med 2010;362:1970-9.

13 Sengupta S, Carrion V, Shelton J, et al. Adverse neonatal outcomes associated with early-term birth. JAMA Pediatr 2013;167:1053-9.

14 Zanardo V, Simbi AK, Franzoi M, et al. Neonatal respiratory morbidity risk and mode of delivery at term: influence of timing of elective caesarean delivery. Acta Paediatr 2004:93:643-7.

15 Zanardo V, Padovani E, Pittini C, et al. The influence of timing of elective cesarean section on risk of neonatal pneumothorax. J Pediatr 2007:150:252-5.

16 Probyn ME, Hooper SB, Dargaville PA, et al. Positive end expiratory pressure during resuscitation of premature lambs rapidly improves blood gases without adversely affecting arterial pressure. Pediatr Res 2004;56:198-204. 